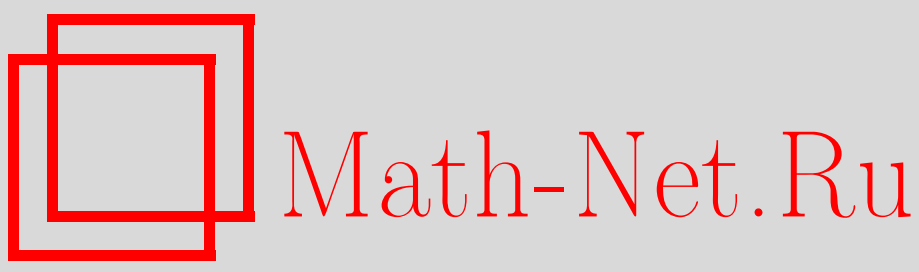

А. Буаве, П. В. Парамонов, Аппроксимация мероморфными и целыми решениями эллиптических уравнений в банаховых пространствах распределений, Матем. сб., 1998, том 189, номер 4, 3-24

DOI: https://doi.org/10.4213/sm303

Использование Общероссийского математического портала Math-Net.Ru подразумевает, что вы прочитали и согласны с пользовательским соглашением

http://www.mathnet.ru/rus/agreement

Параметры загрузки:

IP: 54.197 .217 .227

26 апреля 2023 г., 17:07:12 


\title{
Аппроксимация мероморфными и целыми решениями эллиптических уравнений в банаховых пространствах распределений
}

\begin{abstract}
Для заданного однородного эллиптического дифференциального оператоpa $L$ в частных производных с постоянньми коэффициентами и класса функций (струй-распределений), определенных на замкнутом, не обязательно компактном, подмножестве в $\mathbb{R}^{n}$ и локальн принадлежащих данному банахову пространству $V$, исследуются аппроксимации в норме $V$ функций этого класса цельгми и мероморфными решениями уравнения $L u=0$. Доказываются теоремы типа Рунге, Мергеляна, Рот и Аракеляна для широкого класса банаховых пространств $V$ и операторов $L$, при этом получается большинство известных ранее обобщений этих теорем, а также новые результаты.

Библиографиия: 30 названий.
\end{abstract}

\section{§1. Введение}

Классическая аппроксимационная теорема Рунге [1] общеизвестна. В 1938 г. А. Рот [2] получила следующее обобщение этой теоремы:

Теорема (P). Пусть $F$ - замкнутое (не обязательно компактное) подмножество комплексной плоскости $\mathbb{C}, f$ - голоморфная в (некоторой) окрестности $F$ функиия. Тогда $f$ равномерно на $F$ (с любой точностью) приближается мероморфными функциями с полюсами вне $F$. Если, кроме того, $\overline{\mathbb{C}} \backslash F$ связно и локально связно ( $\overline{\mathbb{C}}-$ сфера Римана), то $f$ равномерно на $F$ приближается цельми функциями.

Один из наиболее сильных результатов в этом направлении принадлежит Н.У. Аракеляну [3]:

Теорема (А). Пусть $F$ - замкнутое подмножество в $\mathbb{C}$. Следующие условия (на $F)$ әквивалентны:

(а) всякая функиия, голоморфная в окрестности $F$, приближается равномерно на $F$ челыми функииями;

(b) всякая функиия, непрерьвная на множестве $F$ и голоморфная в его внутренности $F^{\circ}$, приближсается чельми функциями равномерно на $F$;

(c) множество $\overline{\mathbb{C}} \backslash F$ связно и локально связно.

Работа первого автора поддержана грантом NSERC(Canada).

Работа второго автора выполнена при поддержке Российского фонда фундаменталњных исследований (гранты № 96-01-01240 и № 96-15-96846) и INTAS (грант № 930047). 
Отметим, что для компактов $F$ множество $\overline{\mathbb{C}} \backslash F$ всегда локально связно, так что в этом случае теорема $(\mathrm{P})$ и часть $(\mathrm{c}) \Rightarrow(\mathrm{a})$ теоремы $(\mathrm{A})$ фактически представляют собой утверждения теоремы Рунге, а часть $(\mathrm{b}) \Leftrightarrow(\mathrm{c})$ теоремы $(\mathrm{A})$ есть утверждение известной теоремы С.Н. Мергеляна [4]. В приведенных теоремах аппроксимации осушествляются в стандартной (равномерной) норме пространства $B C(F)$ непрерывных и ограниченных на $F$ функций (при этом сами приближаемые функции не обязательно ограничены), а приближающие функции являются глобальньми (мероморфными и целыми) решениями (эллиптического, в частных производных) уравнения Коши-Римана $\partial f / \partial \bar{z}=0$.

В настоящей работе мы будем рассматривать весьма общие постановки апшроксимационных задач в духе работ [5] и [6]. Наш первый основной результат ( 33 , теорема 1) представляет собой непосредственный аналог торемы (P) (о других ее обобщениях см. [7] и литературу в этой работе). В качестве приближающих функций берутся глобально определенные “L-мероморфные" функции (см. определения в п. 2.3), а классы приближаемых функций (струй-распределений по Уитни) определены на замкнутых (не обязательно компактных) подмножествах $F$ в $\mathbb{R}^{n}$. Аппроксимации осуществляются в банаховых пространствах $(V,\|\cdot\|)$, удовлетворяющих определенным, на наш взгляд весьма естественным, условиям (см. пп. 2.1 и 2.2). Отметим, что доказательство теоремы 1 базируется на локализационной технике, предложенной в [6]. В качестве следствия нашего ( $L$-мероморфного) аналога теоремы (Р) и [6, теорема 3] мы получаем (см. п. 4.2, предложение 1) соответствующую редукционную теорему об аппроксимации для классов функций (названную так, поскольку она "редуцирует", т.е. сводит общую задачу к локальной), которую во многих случаях удается сформулировать в терминах соответствующих емкостей в духе критериев А.Г. Витушкина [8] или даже в чисто геометрических терминах (см. п. 4.2, следствие 2 и п. 4.3, теорема 4 ).

В теореме 3 из $§ 4$ мы приводим точное условие (условие $(\mathrm{N}))$ на пару $(L, V)$, при котором достаточное условие аппроксимации целыми функциями в теореме 1 является одновременно и необходимым, т.е. для этих $(L, V)$ имеет место аналог утверждения $(\mathrm{a}) \Leftrightarrow(\mathrm{c})$ теоремы $(\mathrm{A})$. Последнее позволяет получить также ряд новых аналогов утверждения (b) $\Leftrightarrow(\mathrm{c})$ этой теоремы (см. $\S 4$, теорема 4). В заключение $\S 4$ мы приводим конкретные примеры, а также обсуждаем взаимосвязь между условием $(\mathrm{N})$, порядком банахова пространства $V$ и порядком оператора $L$. Так, в нашу схему укладывается случай равномерных приближений голоморфными функциями [3], но не укладываются равномерные гармонические аппроксимации (см. [9]). Здесь следует упомянуть, что в отличие от большой серии работ, посвященных обобщениям теоремы Рунге (см. [7], [10], [11] и др.), авторам известны всего несколько работ, в которых получены аналоги или обобщения теоремы (А). Приведем их: [12]-[14] (для оператора Коши-Римана и $\operatorname{Lip}_{\alpha}, C^{m}, \mathrm{BMO}$ норм), а также [9] и [15] (для лапласиана и равномерной нормы).

Коротко цель этой работы можно изложить так. Для шширокого класса операторов $L$ и пространств $V$ задачи об аппроксимации функций (струй-распределений) классов $V_{\text {lос }}(F)$ (где $F$ - замкнуто в $\left.\mathbb{R}^{n}\right) L$-мероморфными и $L$-целыми функциями в нормах пространств $V(F)$ (см. п. 2.1) удается единьм методом свести к задачам аппроксимации функций классов $V(K)$ на компактаx $K$ в нормах пространств $V(K)$ функциями $u$, удовлетворяющими уравнению $L u=0$ в (зависящей от $u$ ) окрестности множества $K$. Последняя задача, в свою очередь, уже решена для многих 
конкретных пространств $V$ и операторов $L$ (см. литературу в [16]).

\section{§ 2. Предварительные сведения}

2.1. Банаховы пространства $V$ и $V(F)$. Следуя [6], введем в рассмотрение банахово пространство $V$ с нормой $\|\cdot\|$, которое содержит $C_{0}^{\infty}\left(\mathbb{R}^{n}\right) \equiv C_{0}^{\infty}$ (пространство основных функций в $\left.\mathbb{R}^{n}\right)$ и само содержится в $\left(C_{0}^{\infty}\right)^{*}($ пространстве распределений). Предположим также, что $V$ наделено структурой топологического $C_{0}^{\infty}$-подмодуля в $\left(C_{0}^{\infty}\right)^{*}$. Последнее означает, что при всех $f \in V$ и $\varphi \in C_{0}^{\infty}$ имеет место включение $\varphi f \in V$ (т.е. $V$ полулокально в смысле Хёрмандера [17, определение 2.3.1]), причем

$$
\begin{array}{r}
\|\varphi f\| \leqslant C(\varphi)\|f\|, \\
|\langle f, \varphi\rangle| \leqslant C(\varphi)\|f\|,
\end{array}
$$

где $\langle f, \varphi\rangle$ означает действие распределения $f$ на основную функцию $\varphi$, а константа $C(\varphi)$ не зависит от $f$.

Отметим, что из указанных свойств вытекает непрерывность вложений $C_{0}^{\infty} \hookrightarrow V$ и $V \hookrightarrow\left(C_{0}^{\infty}\right)^{*}$. Действительно, если $\left\|f_{n}\right\| \rightarrow 0, f_{n} \in V$, то из (2) следует, что $\left\langle f_{n}, \varphi\right\rangle \rightarrow 0$ для любого $\varphi \in C_{0}^{\infty}$, т.е. вложение $V \hookrightarrow\left(C_{0}^{\infty}\right)^{*}$ непрерывно в $*$-слабой топологии пространства $\left(C_{0}^{\infty}\right)^{*}$. Далее, если $\varphi_{n} \rightarrow 0$ в $C_{0}^{\infty}$, причем $\left\|\varphi_{n}-\varphi\right\| \rightarrow 0$ в $V$, то $\varphi_{n} \stackrel{w^{*}}{\rightarrow} \varphi$, откуда $\varphi=0$. По теореме о замкнутом графике для LF-пространств [18, гл. 3, теорема 10] вложение $C_{0}^{\infty} \hookrightarrow V$ также является непрерывным. To, что $C_{0}^{\infty}$ является LF-пространством, можно найти, например, в $[19$, гл. $5, \S 4]$.

Для замкнутого множества $F$ в $\mathbb{R}^{n}$ обозначим через $I(F)$ замыкание в $V$ семейства тех элементов $f \in V$, носители которых (рассматриваемые в смысле теории распределений и обозначаемые, соответственно, supp $f$ ) не пересекаются с $F$. Банахово пространство $V(F)=V / I(F)$, наделенное факторнормой, рассматривается нами как наиболее естественная (типа Уитни) версия "сужения" $V$ на $F$ (см. [20, гл. 6]). Через $\|f\|_{F}$ обозначается норма класса эквивалентности (струи) $f_{(F)}:=f+I(F)$ в $V(F)$ распределения $f \in V$.

Для произвольного открытого множества $D$ в $\mathbb{R}^{n}$ положим

$$
V_{\mathrm{loc}}(D)=\left\{f \in\left(C_{0}^{\infty}(D)\right)^{*}: f \varphi \in V \text { для всех } \varphi \in C_{0}^{\infty}(D)\right\} .
$$

При этом $\varphi$ и $f \varphi$ полагаются равными нулю всюду вне $D$.

Через $V_{\mathrm{loc}}$ будем обозначать пространство $V_{\mathrm{loc}}\left(\mathbb{R}^{n}\right)$. В $V_{\text {loc }}(D)$ естественным образом вводится топология Фреше (см. [6, с. 253], где это сделано для случая $V_{\text {loc }}$ ). Для замкнутого множества $F$ определим $V_{\text {loc }}(F)=V_{\text {loc }} / J(F)$, где $J(F)$ есть замы-

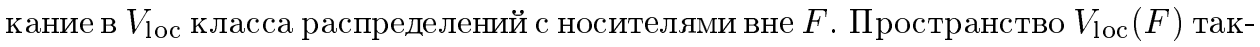
же рассматривается с соответствуюшей фактортопологией (Фреше). Для $f \in V_{\text {loc }}$ положим $f_{(F), \text { loc }}:=f+J(F)$.

Если $D$ является окрестностью $F$, то каждое распределение $h \in V_{\text {loc }}(D)$ естес-

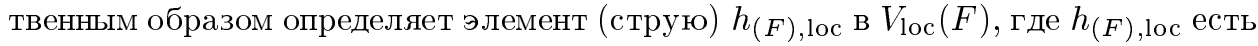
замыкание в $V_{\text {lос }}$ семейства функций $f \in V_{\text {loc }}$, удовлетворяюших условию $f=h$ (как распределения) в некоторой (зависяшей от $f$ ) окрестности $F$. В частности, 
это имеет смысл для всех $h \in C^{\infty}(D) \subset V_{\text {loc }}(D)$. Для $f_{(F) \text {,loc }} \in V_{\text {loc }}(F)$ будем по определению считать, что $f_{(F) \text {, lос }} \in V(F)$ (для краткости пишем $f \in V(F)$ ),

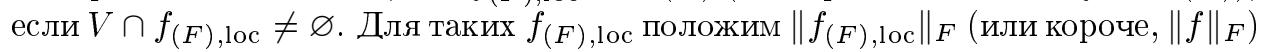
равным $\|g\|_{F}$, где $g \in V \cap f_{(F), \text { loc. }}$.

Докажем, что $\left\|f_{(F), \text { loc }}\right\|_{F}$ определена корректно. Для этого достаточно установить, что равенство

$$
V \cap J(F)=I(F)
$$

справедливо для всех замкнутых множеств $F$. Зафиксируем некоторое локально конечное разбиение единицы $\left\{B_{j}, \varphi_{j}\right\}_{j=1}^{\infty}$ в $\mathbb{R}^{n}$ (здесь $B_{j}-$ шары, $\varphi_{j} \in C_{0}^{\infty}\left(B_{j}\right)$, $\left.\sum_{j=1}^{\infty} \varphi_{j} \equiv 1\right)$ и введем $C_{j}=C\left(\varphi_{j}\right)>0$ - константы из (1) для $\varphi=\varphi_{j}$. Тогда для всякой $f \in V_{\text {lос получим } f \varphi_{j} \in V \text { и }}$

$$
\left\|f \varphi_{j}\right\| \leqslant C_{j}\|f\|_{\bar{B}_{j}}
$$

что непосредственно следует из определения $\|f\|_{\bar{B}_{j}}$. Зафиксируем произвольные замкнутое $F, h \in V \cap J(F)$ и $\varepsilon>0$. Поскольку $h \in J(F)$, то для каждого $j$ можно найти $h_{j} \in V_{\text {loc }}$, удовлетворяюшую условиям $h_{j}=0$ в окрестности $F$ и

$$
\left\|h-h_{j}\right\|_{\bar{B}_{j}}<\frac{\varepsilon}{2^{j} C_{j}}
$$

так что

$$
\left\|h \varphi_{j}-h_{j} \varphi_{j}\right\|<\frac{\varepsilon}{2^{j}} .
$$

Таким образом, распределение $h_{\varepsilon}=\sum_{j=1}^{\infty} h_{j} \varphi_{j}$ принадлежит $V_{\mathrm{loc}}, h_{\varepsilon}=0$ в окрестности $F$ и $\left\|h-h_{\varepsilon}\right\| \leqslant \sum_{j=1}^{\infty}\left\|h \varphi_{j}-h_{j} \varphi_{j}\right\|<\varepsilon$. В частности, $h_{\varepsilon} \in V$, откуда $h_{\varepsilon} \in I(F)$ и, следовательно, $h \in I(F)$.

Полезно отметить также следующий факт. Если $f \in V_{\text {lос }}$ и $f_{(F) \text {,loc }} \in V(F)$, то для любого $\varepsilon>0$ найдется $g_{\varepsilon} \in V$, удовлетворяюшая условиям $f=g_{\varepsilon}$ в некоторой окрестности $F$ и $\left\|g_{\varepsilon}\right\| \leqslant\|f\|_{F}+\varepsilon$. Действительно, так как $f_{(F), \text { lос }} \in V(F)$, то найдется $g \in V$ такая, что $\|g\| \leqslant\|f\|_{F}+\varepsilon / 2$ и $h=f-g \in J(F)$. По аналогии

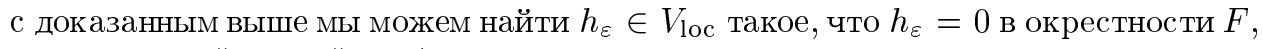
$h_{\varepsilon}-h \in V$ и $\left\|h_{\varepsilon}-h\right\|<\varepsilon / 2$. Искомое $g_{\varepsilon}$ имеет вид $f-h_{\varepsilon}$.

2.2. Эллиптический оператор $L$. Для мультииндекса $\alpha=\left(\alpha_{1}, \ldots, \alpha_{n}\right)$, $0 \leqslant \alpha_{j} \in \mathbb{Z}$, положим $|\alpha|=\alpha_{1}+\cdots+\alpha_{n}, \alpha !=\alpha_{1} ! \cdots \alpha_{n} !, x^{\alpha}=x_{1}^{\alpha_{1}} \cdots x_{n}^{\alpha_{n}}$ при $x=\left(x_{1}, \ldots, x_{n}\right) \in \mathbb{R}^{n}$, а также $\partial^{\alpha}=\left(\partial / \partial x_{1}\right)^{\alpha_{1}} \cdots\left(\partial / \partial x_{n}\right)^{\alpha_{n}}$.

Обозначим через $B(a, \delta)$ (соответственно, $\bar{B}(a, \delta)$ ) открытый (соответственно, замкнутый) шар с центром $a \in \mathbb{R}^{n}$ и радиусом $\delta>0$. Если $B=B(a, \delta)$ и $\theta>0$, то положим $\theta B=B(a, \theta \delta)$ и $\theta \bar{B}=\bar{B}(a, \theta \delta)$.

Всюду в этой работе через $L(\xi)=\sum_{|\alpha|=r} a_{\alpha} \xi^{\alpha}, \xi \in \mathbb{R}^{n}$, обозначается какой-либо фиксированный однородньй полином степени $r$ с постоянными комплексньми коэффициентами, удовлетворяющий условию эллиптичности $L(\xi) \neq 0$ при всех $\xi \neq 0$. Мы ассоциируем с $L(\xi)$ однородный эллиптический оператор $L$ порядка $r$ :

$$
L=L(\partial)=\sum_{|\alpha|=r} a_{\alpha} \partial^{\alpha}
$$


Пусть $\Phi$ - специальное фундаментальное решение для $L$, приведенное, например, в [21] или [6, с. 254], $\varphi \in C_{0}^{\infty}$. Локализационный оператор Витушкина $\mathscr{V}_{\varphi}:\left(C_{0}^{\infty}\right)^{*} \rightarrow\left(C_{0}^{\infty}\right)^{*}$, ассоциированный с $L$ и $\varphi$, определим по формуле $\mathscr{V}_{\varphi} f=$ $\Phi *(\varphi L f)$, в которой “*” означает оператор свертки. Мы потребуем, чтобы для любой функции $\varphi \in C_{0}^{\infty}$ оператор $\mathscr{V}_{\varphi}$ был инвариантным на $V_{\text {loc }}$, т.е. непрерывно отображал $V_{\mathrm{loc}}$ в $V_{\mathrm{loc}}$. Последнее означает, что если $B$ - шар, для которого

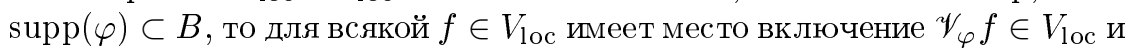

$$
\left\|\mathscr{V}_{\varphi} f\right\|_{\bar{B}} \leqslant C(\varphi, B)\|f\|_{\bar{B}}
$$

где $C(\varphi, B)$ не зависит от $f$.

Отметим, что последнее условие вьполняется для любого (из рассматриваемых) $L$ и всякого пространства $V$, локально эквивалентного какому-либо классическому пространству функций в $\mathbb{R}^{n}$ (см. [5], [6]).

Нам будет необходимо еше одно условие, связываюшее $L$ и $V$. А именно, должны найтись $p \geqslant 0, R_{0}>0$ и положительная функция $\varepsilon(R)$, определенная на $\left[R_{0}, \infty\right)$, $\varepsilon(R) \rightarrow 0$ при $R \rightarrow \infty$, такие, что для любых $R \geqslant R_{0}$ и $\alpha \in \mathbb{Z}_{+}^{n},|\alpha| \geqslant p$, справедлива оценка

$$
\left\|\partial^{\alpha} \Phi(x)\right\|_{\mathbb{R}^{n} \backslash B(0, R)} \leqslant \alpha !(\varepsilon(R))^{|\alpha|} .
$$

$\mathrm{B}$ частности, $\partial^{\alpha} \Phi \in V\left(\mathbb{R}^{n} \backslash B(0, R)\right)$ при $|\alpha| \geqslant p$. Ряд примеров, связанных с условием (4), см. в [6, с. 253-254]. Из условий (1)-(4) вытекает "специальный принцип максимума", сформулированньй в лемме 1 (он и будет использоваться вместо условия (4)).

2.3. $L$-аналитические и $L$-мероморфные функции. Пусть $D$ - открытое множество в $\mathbb{R}^{n}$. Обозначим через $L(D)$ класс распределений $f$ в $D$, для которых $L f=0$ в $D$ в смысле теории распределений. Хорошо известно (лемма Вейля), что $L(D) \hookrightarrow C^{\infty}(D)$, откуда $L(D) \subset V_{\text {loc }}(D)$. Далее, если $\left\{f_{m}\right\}$ - последовательность в $L(D)$ такая, что $f_{m} \rightarrow f$ в $V_{\text {loc }}(D)$ при $m \rightarrow \infty$, то $f \in L(D)$, поскольку сходимость в $V_{\text {loc }}(D)$ сильнее сходимости в $\left(C_{0}^{\infty}\right)^{*}$, а для нее последнее утверждение выполняется. Функции из $L(D)$ и $L\left(\mathbb{R}^{n}\right)$ будем назьвать $L$-аналитическими в $D$ и $L$-целыми, соответственно.

Будем говорить, что функция $g$ является $L$-мероморфной в $\mathbb{R}^{n}$, если $\operatorname{supp}(L g)$ дискретен, а для любой $a \in \operatorname{supp}(L g)$ найдутся $h, L$-аналитическая в окрестности точки $a$, и $\lambda_{\alpha} \in \mathbb{C}, 0 \leqslant|\alpha| \leqslant k<\infty$, такие, что

$$
g(x)=h(x)+\sum_{|\alpha| \leqslant k} \lambda_{\alpha} \partial^{\alpha} \Phi(x-a)
$$

в некоторой окрестности точки $a$. Точки $a \in \operatorname{supp}(L g)$ будут называться полюсами функции $g$.

2.4. Ряды Тейлора и Лорана. Пусть $B=B(a, \delta), g \in L(B)$. Хорошо известно, что $g$ вешественно-аналитична в $B$ и, кроме того, найдется $\theta_{1} \in(0,1)$, зависящая только от $L$, такая, что

$$
g(x)=\sum_{|\alpha| \geqslant 0} \frac{\partial^{\alpha} g(a)}{\alpha !}(x-a)^{\alpha}, \quad x \in \theta_{1} B,
$$


причем этот ряд сходится в $C^{\infty}\left(\theta_{1} B\right)$. Заметим, что однородные составляющие ряда (5) являются $L$-аналитическими полиномами. В самом деле, положим

$$
P_{m}(x-a)=\sum_{|\alpha|=m} \frac{\partial^{\alpha} g(a)}{\alpha !}(x-a)^{\alpha} .
$$

Пусть $p$ - степень многочлена $L P_{m}$ и $\beta$ - мультииндекс ранга $|\beta|=p$. В шаре $\theta_{1} B$ вьполнено

$$
0=L\left(\partial^{\beta} g\right)=L\left(\sum_{j} \partial^{\beta} P_{j}\right)=\sum_{j} \partial^{\beta} L P_{j}=\left(\partial^{\beta} L P_{m}\right)(0)+\cdots
$$

где многоточием обозначены слагаемые высших порядков. Согласно выбору $\beta$ $\partial^{\beta} L P_{m}$ есть константа, т.е. $\partial^{\beta}\left(L P_{m}\right)=0$ при $|\beta|=p$. Следовательно, $L P_{m} \equiv 0$.

Напомним, что вложение $C_{0}^{\infty} \hookrightarrow V$ непрерывно. Отсюда и из (5) следует, что для каждого $\theta \in\left(0, \theta_{1}\right)$ и любого положительного $\varepsilon$ найдется $L$-аналитический полином $P_{\varepsilon}$ такой, что

$$
\left\|g-P_{\varepsilon}\right\|_{\theta \bar{B}}<\varepsilon
$$

Напомним также (см. [6], [7, с. 239]), что найдется $k>1$ такое, что если $T-$ распределение с компактным носителем в $B(a, \delta)$ и $f=\Phi * T$, то при $|x-a|>k \delta$ справедливо разложение лорановского типа:

$$
f(x)=\langle T(y), \Phi(x-y)\rangle=\sum_{|\alpha| \geqslant 0} c_{\alpha} \partial^{\alpha} \Phi(x-a)
$$

где $c_{\alpha}=(-1)^{|\alpha|}(\alpha !)^{-1}\left\langle T(y),(y-a)^{\alpha}\right\rangle$, причем этот ряд сходится в смысле $C^{\infty}(|x-a|>k \delta)$.

\section{§3. Теорема типа Рунге и достаточные условия аппроксимации $L$-целыми функциями}

3.1. Теорема типа Рунге. Обозначим через $\mathbb{R}_{\infty}^{n}=\mathbb{R}^{n} \cup\{\infty\}$ одноточечную компактификацию $\mathbb{R}^{n}$. Замкнутое подмножество $F$ в $\mathbb{R}^{n}$ назовем РКЛ-мно жеством, если $\mathbb{R}_{\infty}^{n} \backslash F$ является связньп и локально связным. Впервые такие множества (в контексте теории приближений) были введены в работах Рот [2], М. В. Келдыша и М.А. Лаврентьева [22], однако в литературе их часто именуют К-множествами (подробнее об этих множествах см. [23, гл.4], а также $\$ 4$, замечание 4).

Теперь мы готовы сформулировать наш первый основной результат - аналог теоремы (Р) $\S 1$ об аппроксимациях $L$-мероморфными и $L$-целыми функциями.

ТЕОРема 1. Пусть пара $(L, V)$ удовлетворяет условиям (1)-(4), F - замкнутое подмножество в $\mathbb{R}^{n}$ и $f$ является $L$-аналитической в окрестности $F$. Тогда для всякого $\varepsilon>0$ существует $L$-мероморфная функиия $g$ с полюсами вне $F$ такая, что $\left(f_{(F), \text { loc }}-g_{(F), \text { loc }}\right) \in V(F)$ u

$$
\|f-g\|_{F}<\varepsilon \text {. }
$$

Более того, если F - РКЛ-множество, то в качестве $g$ может бъть въбрана L-целая функиия.

Непосредственным следствием теоремы 1 и [6, теорема 3] является 
ТЕОРема 2. Пусть пара $(L, V)$ удовлетворяет условиям (1)-(4), F - замкнуто в $\mathbb{R}^{n}, f \in V_{\mathrm{loc}}$. Следующие утвержсдения әквивалентны:

(i) для произвольного положительного в найдется L-мероморфная функиия $g$ с полюсами вне $F$ такая, что $\left(f_{(F), \text { loc }}-g_{(F), \mathrm{loc}}\right) \in V(F)$ u $\|f-g\|_{F}<\varepsilon$

(ii) для любого шара $B$ и положстельного в найдется функиия $g$ такая, что $L g=0$ в некоторой окрестности $F \cap \bar{B} u\|f-g\|_{F \cap \bar{B}}<\varepsilon$;

(iii) предыдущее утвержсдеие верно для каждого иара из некоторого локально конечного семейства шаров $\left\{B_{j}^{\prime}\right\}$, покрывающего $F$.

Уточним, что при получении (iii) $\Rightarrow$ (i) последней теоремы мы модифицируем доказательство теоремы 3 из [6, с. 257] следующим образом. В самом начале этого доказательства вместо семейства конгруэнтных шаров $\left\{B_{j}\right\}$ мы выбираем такое локально конечное покрытие $\left\{B_{j}\right\}$ всего $\mathbb{R}^{n}$ (и соответствующее подчиненное ему разбиение единицы $\left.\left\{\varphi_{j}\right\}\right)$, что при каждом $j$ выполняется $\bar{B}_{j} \cap F=\varnothing$ или $\bar{B}_{j} \subset B_{s}^{\prime}$ при некотором $s$. Остальная часть доказательства остается без изменений.

ЗАмечАнИЕ 1. Из теоремы 2 нетрудно получить соответствующую аппроксимационную теорему для классов функций (струй) (см. п. 4.2, предложение 1). Во многих конкретных случаях ее удается установить в терминах соответствующих емкостей и (или) обхватов по Хаусдорфу как аналог теорем Витушкина [8] и Нерсесяна [24] (см. п. 4.3, теорема 4).

ЗАмечАниЕ 2. Следует отметить, что приближения, имеющие место в теореме 1 и теореме 2 (i), вообще говоря, могут быть более сильными, чем в топологии (Фреше) пространства $V_{\text {loc }}(F)$. С другой стороны, в теореме 2 утверждается, что при соответствующих условиях эти типы приближений ((i) и (ii) в теореме 2) эквивалентны. Далее, условие (4) в теоремах 1 и 2 можно отбросить, если $F$ - компакт.

ЗАмечАниЕ 3 . Для широкого класса пар $(L, V)$ условие на $F$ быть РКЛ-множеством является не только достаточным, но также и необходимым для возможности приближения всякой $L$-аналитической в окрестности $F$ функции $L$-цельммu функциями в норме $V(F)$. Для таких $(L, V)$, подпадающих, кроме того, под описание в замечании 1 , удается получить (п. 4.3, теорема 4 ) полную характеризацию $L V$-множеств Аракеляна, т.е. обобщить на эти случаи основные результаты работ [3], [12]-[14] (в которых $L=\bar{\partial}=\partial / \partial \bar{z}$ - оператор Коши-Римана в $\mathbb{C}$ ).

3.2. Доказательство теоремы 1. Из условий (1)-(4) вытекает следующая лемма, доказанная в [6].

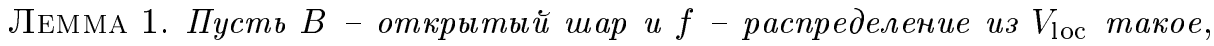
что $L f=0$ в $\mathbb{R}^{n} \backslash B$ u $f(x)=O\left(|x|^{-d}\right)$ nри $x \rightarrow \infty$, әде $d=\max \{p+n-r ; 1\}, p-$ челое число из условия (4). Тогда $f \in V u\|f\| \leqslant C\|f\|_{3 \bar{B}}$, где $C$ - константа, не зависящая от $f$.

Следуюшее утверждение использовалось без доказательства в [6] при получении предыдушей леммы. Для полноты изложения мы предпочли привести это доказательство, тем более что оно с минимальными изменениями подойдет и для леммы 5. 
Лемма 2. Пусть $V$ - банахово пространство с условием (1). Тогда для всякого открытого шара $B$ в $\mathbb{R}^{n}$ найдется константа $A=A(V, B)>0$ такая, что из условий $f \in V_{\mathrm{loc}} u f \in V\left(\mathbb{R}^{n} \backslash B\right)$ вытекает, что $f \in V$ $u$

$$
\|f\| \leqslant A\left(\|f\|_{2 \bar{B}}+\|f\|_{\mathbb{R}^{n} \backslash B}\right) .
$$

ДокАЗАТЕЛЬСтво. Выберем $\varphi \in C_{0}^{\infty}(2 B), \varphi=1$ в некоторой окрестности шара $\bar{B}$, и положим $f_{1}=\varphi f, f_{2}=(1-\varphi) f=f-\varphi f$. Ясно, что $f_{1} \in V$. Покажем, что $f_{2}$ тоже принадлежит $V$. Действительно, поскольку $f \in V\left(\mathbb{R}^{n} \backslash B\right)$, найдется $g \in V$ такая, что $g=f$ в окрестности $\mathbb{R}^{n} \backslash B$ и $\|g\| \leqslant 2\|f\|_{\mathbb{R}^{n} \backslash B}$ (см. п. 2.1). Следовательно, $f_{2}=(1-\varphi) g=g-\varphi g \in V$, что и требовалось.

Теперь заметим, что $f_{1}$ на самом деле определяется также и элементом $f_{(2 \bar{B})}$. Поэтому из определения элемента $f_{(2 \bar{B})}$ мы можем найти $h \in V$ такую, что $h_{(2 \bar{B})}=$ $f_{(2 \bar{B})}$ и $\|h\| \leqslant 2\|f\|_{2 \bar{B}}$. Таким образом, $f_{1}=\varphi h$. Из (1) получаем

$$
\left\|f_{1}\right\| \leqslant C(\varphi)\|h\| \leqslant A_{1} C(\varphi)\|f\|_{2} \bar{B} .
$$

По аналогии,

$$
\left\|f_{2}\right\|=\|(1-\varphi) g\| \leqslant\|g\|+C(\varphi)\|g\| \leqslant A_{2}(1+C(\varphi))\|f\|_{\mathbb{R}^{n} \backslash B} .
$$

Остается вспомнить, что $f=f_{1}+f_{2}$. Лемма доказана.

Лемма 3. Пусть $(L, V)$ удовлетворяет условиям (1)-(4), T-распределенuе, $\operatorname{supp} T \subset B(a, \delta), f=\Phi * T u$

$$
f_{m}=\sum_{0 \leqslant|\alpha| \leqslant m} c_{\alpha} \partial^{\alpha} \Phi(x-a)
$$

- частичные суммы разложсения Лорана функиии $f$ вне $\bar{B}(a, k \delta)$ (см. п. 2.4). Тогда найдется такое натуральное $M$, что для всех $m>M$ имеем $f-f_{m} \in$ $V\left(\mathbb{R}^{n} \backslash B(a, 2 k \delta)\right) u$

$$
\left\|f-f_{m}\right\|_{\mathbb{R}^{n} \backslash B(a, 2 k \delta)} \rightarrow 0 \quad \text { npu } \quad m \rightarrow \infty .
$$

ДоказАтельство. Сначала напомним, что $f_{m} \rightarrow f$ в $C^{\infty}\left(\mathbb{R}^{n} \backslash \bar{B}(a, k \delta)\right)$. Пусть $\psi \in C^{\infty}\left(\mathbb{R}^{n}\right)$ таково, что

$$
\psi= \begin{cases}0 & \text { в окрестности } \bar{B}(a, k \delta), \\ 1 & \text { в окрестности } \mathbb{R}^{n} \backslash B(a, 2 k \delta) .\end{cases}
$$

Имеем $\psi f_{m} \rightarrow \psi f$ в $C^{\infty}\left(\mathbb{R}^{n}\right)$ и

$$
\left\|f_{m}-f\right\|_{\mathbb{R}^{n} \backslash B(a, 2 k \delta)} \leqslant\left\|\psi\left(f_{m}-f\right)\right\| .
$$

Кроме того, заметим, что для достаточно больших $m$ распределение $\psi\left(f_{m}-f\right)$ удовлетворяет условиям леммы 1 , откуда $\psi\left(f_{m}-f\right) \in V$ и

$$
\left\|\psi\left(f_{m}-f\right)\right\| \leqslant C\left\|\psi\left(f_{m}-f\right)\right\|_{\bar{B}(a, 6 k \delta)} .
$$

Для завершения доказательства леммы 3 остается учесть, что

$$
\left\|\psi\left(f_{m}-f\right)\right\|_{\bar{B}(a, 6 k \delta)} \rightarrow 0 \text { при } m \rightarrow \infty,
$$

так как топология в $C^{\infty}$ сильнее, чем в $V_{\text {loc }}$. 
ДОКАЗАТЕЛЬСТВо ТЕОРЕМЫ 1 . Пусть $f$ является $L$-аналитической в окрестности $U$ множества $F$. Пусть $U_{1}$ - такая окрестность множества $F$, что $\bar{U}_{1} \subset U$. Продолжим $f$ до функции (также обозначаемой через $f$ ) из $C^{\infty}\left(\mathbb{R}^{n}\right)$ так, чтобы она осталась $L$-аналитической в окрестности множества $\bar{U}_{1}$. Напомним, что $\theta$ и $k$ - константы из п. 2.4. Найдем последовательность пар $\left\{B\left(a_{j}, \delta_{j}\right), \varphi_{j}\right\}_{j=1}^{\infty}$, где семейство шаров $\left\{B_{j}\right\}=\left\{B\left(a_{j}, \delta_{j}\right\}\right.$ локально конечно, $2 k \bar{B}_{j} \cap F=\varnothing, \varphi_{j} \in C_{0}^{\infty}\left(B_{j}\right)$, $0 \leqslant \varphi_{j} \leqslant 1$, причем $\sum_{j=1}^{\infty} \varphi_{j}=1$ в некоторой окрестности $U_{2}$ множества $\mathbb{R}^{n} \backslash U_{1}$.

Определим $f_{j}=\mathscr{V}_{\varphi_{j}} f=\Phi *\left(\varphi_{j} L f\right)$. Каждое $f_{j}$ принадлежит $C^{\infty}\left(\mathbb{R}^{n}\right)$. При $l \geqslant 1$ положим $J_{l}=\left\{j: B_{j} \cap B(0, l / \theta) \neq \varnothing\right\}$. Отметим, что

$$
L\left(f-\sum_{j \in J_{1}} f_{j}\right)=L f-\sum_{j \in J_{1}} \varphi_{j} L f=L f\left(1-\sum_{j \in J_{1}} \varphi_{j}\right)=0
$$

(т.е. $f-\sum_{j \in J_{1}} f_{j}$ является $L$-аналитической) в $B(0,1 / \theta)$. Следовательно (см. (6)), найдется $L$-аналитический полином $P_{1}$ такой, что

$$
\left\|f-\left(\sum_{J_{1}} f_{j}\right)-P_{1}\right\|_{\bar{B}(0,1)}<\frac{1}{2}
$$

Далее, поскольку $f-\left(\sum_{J_{1}} f_{j}\right)-P_{1}-\left(\sum_{J_{2} \backslash J_{1}} f_{j}\right)$ является $L$-аналитической в $B(0,2 / \theta)$, найдется такой $L$-аналитический полином $P_{2}$, что

$$
\left\|f-\left(\sum_{J_{1}} f_{j}\right)-P_{1}-\left(\sum_{J_{2} \backslash J_{1}} f_{j}\right)-P_{2}\right\|_{\bar{B}(0,2)}<\frac{1}{2^{2}}
$$

По индукции найдем такие $L$-аналитические полиномы $P_{l}$, что

$$
\left\|f-\left(\sum_{J_{1}} f_{j}\right)-P_{1}-\left(\sum_{J_{2} \backslash J_{1}} f_{j}\right)-P_{2}-\ldots-\left(\sum_{J_{l} \backslash J_{l-1}} f_{j}\right)-P_{l}\right\|_{\bar{B}(0, l)}<\frac{1}{2^{l}},
$$

так что равенство

$$
f=\sum_{l=1}^{\infty}\left(\sum_{J_{l} \backslash J_{l-1}} f_{j}+P_{l}\right)
$$

справедливо в $V_{\text {loc }}$.

Каждое $f_{j}$ разложим в ряд типа Лорана (см. п. 2.4)

$$
f_{j}(x)=\sum_{|\alpha| \geqslant 0} c_{\alpha}^{j} \partial^{\alpha} \Phi\left(x-a_{j}\right)
$$

сходящийся вне $k \bar{B}_{j}$ и, следовательно, в окрестности $F$. По лемме 3 для каждого $\eta_{j}>0$ найдется $m_{j}$ такое, что для

$$
\begin{array}{r}
g_{j}(x)=\sum_{|\alpha|=0}^{m_{j}} c_{\alpha}^{j} \partial^{\alpha} \Phi\left(x-a_{j}\right) \\
\text { имеем }\left(f_{j}-g_{j}\right) \in V\left(\mathbb{R}^{n} \backslash 2 k B_{j}\right) \text { и }\left\|g_{j}-f_{j}\right\|_{\mathbb{R}^{n} \backslash 2 k B_{j}}<\eta_{j} .
\end{array}
$$


Положим $F_{1}=\mathbb{R}^{n} \backslash \bigcup_{j}\left(2 k B_{j}\right)$. Тогда $F \subset F_{1}$ и для всех $j$ справедливо $\left(f_{j}-g_{j}\right) \in V\left(F_{1}\right)$ и $\left\|f_{j}-g_{j}\right\|_{F_{1}}<\eta_{j}$. Зафиксируем $\varepsilon>0$ и выберем последовательность $\left\{\eta_{j}\right\}, \eta_{j}>0$, так, чтобы $\sum_{j} \eta_{j}<\varepsilon$. Обозначим

$$
g=\sum_{l=1}^{\infty}\left(\sum_{J_{l} \backslash J_{l-1}} g_{j}+P_{l}\right)
$$

где $J_{0}=\varnothing$.

Поскольку для каждого $m \geqslant 1$ ряд

$$
\sum_{l=m+1}^{+\infty}\left(\sum_{J_{l} \backslash J_{l-1}} g_{j}+P_{l}\right)
$$

сходится в $V(\bar{B}(0, m))$, то $g$ есть $L$-мероморфная функция с полюсами (возможно только) в $a_{j}, j=1,2, \ldots$

Более того, $g \in V_{\mathrm{loc}}\left(F_{1}\right)$ и

$$
(f-g)_{\left(F_{1}\right), \mathrm{loc}}=\sum_{l=1}^{\infty}\left(\sum_{J_{l} \backslash J_{l-1}}\left(f_{j}-g_{j}\right)_{\left(F_{1}\right), \mathrm{loc}}\right) .
$$

Но тогда $f-g \in V(F)$ и

$$
\|f-g\|_{F}<\varepsilon,
$$

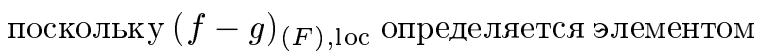

$$
\sum_{l=1}^{\infty}\left(\sum_{J_{l} \backslash J_{l-1}} \Psi_{j}\right)
$$

где $\Psi_{j} \in V$ таковы, что $\left(\Psi_{j}\right)_{\left.\mathbb{R}^{n} \backslash 2 k B_{j}\right)}=\left(f_{j}-g_{j}\right)_{\left(\mathbb{R}^{n} \backslash 2 k B_{j}\right)}$ и $\left\|\Psi_{j}\right\| \leqslant \eta_{j}$. Первая часть теоремы 1 доказана.

Предположим теперь, что $F$ есть РКЛ-множество, т.е. $\mathbb{R}_{\infty}^{n} \backslash F$ связно и локально связно. Мы должны найти $L$-целую функцию $h \in V_{\text {lос такую, что }}$

$$
\|g-h\|_{F}<\varepsilon .
$$

Пусть $\left\{a_{j}\right\}_{j \geqslant 1}$ - последовательность полюсов функции $g$. Она лежит в $\mathbb{R}^{n} \backslash F$ и не имеет предельных точек в $\mathbb{R}^{n}$. Поскольку $\mathbb{R}_{\infty}^{n} \backslash F$ связно и локально связно в $\infty$, найдутся пути $\sigma_{j}$, соединяюшие $a_{j}$ и $\infty, \sigma_{j} \subset \mathbb{R}^{n} \backslash F$, причем семейство $\left\{\sigma_{j}\right\}$ локально конечно в $\mathbb{R}^{n}$.

Зафиксируем $j$ и для $m=0,1, \ldots$ построим $B_{j_{m}}=B\left(a_{j_{m}}, 2 k r_{j_{m}}\right) \subset \mathbb{R}^{n} \backslash F$ такие, что $a_{j_{m}} \in \sigma_{j}, a_{j_{0}}=a_{j}, a_{j_{m}} \rightarrow \infty$ при $m \rightarrow \infty,\left|a_{j_{m}}-a_{j_{m+1}}\right|<r_{j_{m+1}}<1$, семейство шаров $B_{j_{m}}$ локально конечно в $\mathbb{R}^{n}$, причем $\bar{G}_{j} \cap F=\varnothing$, где $G_{j}=$ $\bigcup_{m=0}^{\infty} B_{j_{m}}$. Мы можем выбрать эти шары так, что $\left\{G_{j}\right\}$ тоже локально конечно в $\mathbb{R}^{n}$. 
Положим $h_{0}=g$. Построим последовательность функций $h_{j}, L$-мероморфных в $\mathbb{R}^{n}$, так, чтобы при каждом $j=1,2, \ldots$ функция $h_{j}$ имела те же полюсы (и сингулярные части), что и $h_{j-1}$, кроме точки $a_{j}$, где $h_{j}$ является $L$-аналитической, причем

$$
\left\|h_{j-1}-h_{j}\right\|_{\mathbb{R}^{n} \backslash G_{j}}<\frac{\varepsilon}{2^{j}} .
$$

Если такая последовательность найдена, то $h=\lim _{j \rightarrow \infty} h_{j}$ есть $L$-целая функция. Действительно, по построению (напомним, что $\left\{G_{j}\right\}$ локально конечно) имеем $G_{j} \rightarrow \infty$ при $j \rightarrow \infty$, поэтому $\left\{h_{j}\right\}$ есть последовательность Коши в $V(\bar{B}(0, R))$ при любом $R>0$, остается воспользоваться тем (см. п. 2.3), что сходимость в $V_{\text {lос }}$ сохраняет $L$-аналитичность. Более того, мы имеем $\|g-h\|_{F}<\varepsilon$, что и требовалось.

Для построения $h_{j}$ положим $h_{0}=g$ и допустим, что $h_{l}$ уже построены для $l \leqslant j-1$. Пусть $s_{0}-$ сингулярная часть функции $h_{j-1}$ в точке $a_{j}=a_{j_{0}}$. По лемме 3 найдется $L$-мероморфная функция $s_{1}$ с единственной особой точкой $a_{j_{1}}$, удовлетворяюшая условию

$$
\left\|s_{0}-s_{1}\right\|_{\mathbb{R}^{n} \backslash B_{j_{1}}}<\frac{1}{2} \cdot \frac{\varepsilon}{2^{j}} .
$$

По индукции построим $L$-мероморфную функцию $s_{m}$ с единственной особенностью в $a_{j_{m}}$, удовлетворяюшую условию

$$
\left\|s_{m-1}-s_{m}\right\|_{\mathbb{R}^{n} \backslash B_{j m}}<\frac{1}{2^{m}} \cdot \frac{\varepsilon}{2^{j}} .
$$

Наконец, положим

$$
h_{j}=h_{j-1}+\sum_{m=1}^{\infty}\left(s_{m}-s_{m-1}\right) .
$$

Функция $h_{j}$ - искомая. Теорема 1 доказана.

\section{§4. О необходимых условиях приближаемости $L$-целыми функциями}

4.1. Основной результат и его доказательство. В пा. 4.1 и 4.2 мы будем предполагать (или проверять), что наше пространство $V$ и оператор $L$ (с фундаментальньм решением $\Phi$ в $\mathbb{R}^{n}$ ) удовлетворяют условиям (1) - (4), приведенньм в $\S 2$.

Для замкнутого множества $F$ в $\mathbb{R}^{n}$ обозначим через $M_{L V}(F)$ (соответственно, $\left.E_{L V}(F)\right)$ класс всех $f_{(F), \text { loc }} \in V_{\text {loc }}(F)$, которые удовлетворяют следуюшему условию: для каждого $\varepsilon>0$ найдется функция $g, L$-мероморфная в $\mathbb{R}^{n}$ с полюсами вне $F$ (соответственно, $L$-целая), такая, что $f-g \in V(F)$ и $\|f-g\|_{F}<\varepsilon$. Введем еше пространство $V_{L}(F)=V_{\text {loc }}(F) \cap L\left(F^{\circ}\right)$, где $F^{\circ}$ - внутренность множества $F$. По теореме $1 M_{L V}(F)$ есть замыканиев $V_{\text {loc }}(F)$ пространства $\left\{h_{(F), \text { loc }} \in V_{\text {loc }}(F)\right.$ : $h$ является $L$-аналитической в некоторой (зависящей от $h$ ) окрестности $F\}$. Более того, если $F$ есть РКЛ-множество, то $M_{L V}(F)=E_{L V}(F)$.

Множества $F$, для которых $V_{L}(F)=E_{L V}(F)$, будем называть $L V$-множествами Аракеляна. 
ЗАмечАниЕ 4 . Напомним, что замкнутое множество $F$ в $\mathbb{R}^{n}$ является РКЛ-множеством (т.е. множество $G=\mathbb{R}_{\infty}^{n} \backslash F$ связно и локально связно), если и только если для каждого $R>0$ найдется $R^{\prime}>R$, удовлетворяющее следующему условию: для всякой точки $a \in G, R^{\prime}<|a|<+\infty$, существует непрерывное отображение $\gamma:[0,+\infty) \rightarrow G$ такое, что $\gamma(0)=a,|\gamma(t)| \rightarrow+\infty$ при $t \rightarrow+\infty$ и $|\gamma(t)|>R$ при всех $t \geqslant 0$. Более того, для компактов $F$ в $\mathbb{R}^{n}$ пространство $E_{L V}(F)$ совпадает с $P_{L V}(F)$ - замыканием в $V(F)$ пространства $\left\{p_{(F)}: p\right.$ является $L$-аналитическим полиномом $\}$, причем для таких $F$ множество $G=\mathbb{R}_{\infty}^{n} \backslash F$ всегда локально связно.

Пусть $K$ - компакт в $\mathbb{R}^{n}$. Обозначим через $\widehat{K}$ объединение $K$ и всех его ограниченных компонент (связности) дополнения. Очевидно, что условие $\widehat{K}=K$ эквивалентно связности дополнения компакта $K$. Положим

$$
N(K)=\left\{a \in \widehat{K} \backslash K:\left(\Phi_{a}\right)_{(K)} \notin P_{L V}(K)\right\}
$$

где $\Phi_{a}(x)=\Phi(x-a)$.

УСловИЕ $(\mathrm{N})$. Будем говорить, что пара $(L, V)$ удовлетворяет условию $(\mathrm{N})$ ("незатираемости дьр"), если $N(K) \neq \varnothing$ для всякого компакта $K$ с “дырами”, т.е. такого, что $K \neq \widehat{K}$.

Второй основной результат этой работы состоит в следующем.

ТЕОРема 3. Пусть $(L, V)$ удовлетворяет условиям (1)-(4). Тогда следующие утверждения равносильны:

(i) для всякого замкнутого множества $F \subset \mathbb{R}^{n}$

$$
M_{L V}(F)=E_{L V}(F) \Longleftrightarrow\{F \text { есть РКЛ-мноэсество }\}
$$

(ii) для любого компакта $K \subset \mathbb{R}^{n}$

$$
M_{L V}(K)=P_{L V}(K) \Longleftrightarrow\left\{\mathbb{R}^{n} \backslash K \text { связно }\right\}
$$

(iii) пара $(L, V)$ удовлетворяет условию $(\mathrm{N})$.

Таким образом, условие $(\mathrm{N})$ в точности описывает пары $(L, V)$ (со свойствами $(1)-(4))$, для которых справедлив аналог утверждения (a) $\Leftrightarrow(c)$ теоремы (A). Важно также отметить, что в условии $(\mathrm{N})$ по определению рассматриваются только компактные множества $K$. Если ограничиться компактами $F$, то условия $(3)$ и (4) в формулировке последней теоремы можно отбросить.

В качестве приложений теоремы 3 мы получаем аналоги утверждения (b) $\Leftrightarrow(\mathrm{c})$ теоремы (А) для большого класса пар $(L, V)$ (см. п. 4.3, теорема 4$)$.

В доказательстве теоремы 3 используются две следующие леммы, в которых условие $(\mathrm{N})$ не требуется.

Лемма 4. Пусть $K$ - компакт в $\mathbb{R}^{n}$ с условием $N(K) \neq \varnothing$ (в частности, $K \neq \widehat{K})$. Пусть $D$ - такая ограниченная компонента из $\widehat{K} \backslash K$, что $N_{D}(K)=$ $N(K) \cap D \neq \varnothing$. Тогда $N_{D}(K)$ открыто и всюду плотно в $D$. 
ДокаЗАТЕЛЬСтво. Пусть, от противного, $N_{D}(K)$ не является открытым, т.е. найдется $a \in N_{D}(K)$ такое, что $a_{l} \rightarrow a$ при $l \rightarrow+\infty$ для некоторой последовательности $\left\{a_{l}\right\} \subset D \backslash N_{D}(K)$. Тогда найдется такая окрестность $U$ множества $K$, что $\Phi_{a_{l}} \rightarrow \Phi_{a}$ равномерно на $\bar{U}$ при $l \rightarrow+\infty$. При этом $\Phi_{a_{l}} \rightarrow \Phi_{a}$ также и в пространстве $C^{\infty}(U)$ и, следовательно, в $V(K)$ при $l \rightarrow+\infty$. Таким образом, $\Phi_{a} \in P_{L V}(K)$. Противоречие.

Если, от противного, $N_{D}(K)$ не плотно в $D$, то найдется шар $B \subset D \backslash N(K)$, в котором $\Phi_{b} \in P_{L V}(K)$ для каждого $b \in B$. По определению частных производных имеем

$$
\frac{\partial}{\partial x_{i}} \Phi_{b}(x)=\lim _{\Delta x_{i} \rightarrow 0} \frac{\Phi\left(x+\Delta x_{i} e_{i}-b\right)-\Phi(x-b)}{\Delta x_{i}}=\lim _{\Delta x_{i} \rightarrow 0} \frac{\Phi_{b-\Delta x_{i} e_{i}}(x)-\Phi_{b}(x)}{\Delta x_{i}},
$$

где $x \neq b, i=1, \ldots, n,\left\{e_{i}\right\}_{i=1}^{n}-$ стандартньй базис в $\mathbb{R}_{x}^{n}$. Указанньй предел является равномерньм на каждом компакте в $\mathbb{R}^{n} \backslash\{b\}$, и следовательно (см. выше), этот предел сушествует также в $V(K)$.

По индукции получим, что $\partial^{\alpha} \Phi_{b} \in P_{L V}(K)$ для всех $\alpha \in \mathbb{Z}_{+}^{n}$ и $b \in B$. Используя разложения типа Лорана и метод Рунге (см. доказательство теоремы 1$)$, нетрудно установить, что множество $\left\{b \in D: \partial^{\alpha} \Phi_{b} \in P_{L V}(K)\right.$ при всех $\left.\alpha \in \mathbb{Z}_{+}^{n}\right\}$ одновременно открыто и замкнуто в $D$, т.е. совпадает с $D$. Последнее противоречит предположению $N_{D}(K) \neq \varnothing$. Лемма 4 доказана.

Лемма 5. Для каждого шара $B$ в $\mathbb{R}^{n}$ найдется константа $A=A(V, B) \in$ $(0,+\infty)$ такая, ито для любого компакта $K$ и любой $f_{(K)} \in V(K)$ имеем

$$
\|f\|_{K} \leqslant A\left(\|f\|_{K \cap 3 \bar{B}}+\|f\|_{K \backslash(2 B)}\right) .
$$

ДокаЗАтЕльство. Аналогично доказательству леммы 2. Заметим только, что каждый элемент $f_{(K)}$ из $V(K)$ естественно определяет элемент $f_{\left(K^{\prime}\right)} \in V\left(K^{\prime}\right)$ для любого компакта $K^{\prime} \subset K$.

ДокаЗАТЕЛЬСТво ТЕОРЕмы 3. Очевидно, что (i) $\Rightarrow$ (ii). Установим (ii) $\Rightarrow$ (iii). Пусть (ii) верно и $K$ - произвольньй компакт, для которого $\widehat{K} \neq K$. Из (ii) вытекает, что найдется окрестность $U$ компакта $K$ и $f \in L(U) \backslash P_{L V}(K)$. Мы можем дополнительно предположить, что $f \in C_{0}^{\infty}$. Согласно [6, лемма 1] имеем $f=\Phi *(L f)$. Пользуясь тем, что римановы суммы интеграла из предыдушей свертки сходятся $\mathrm{K}$ нему равномерно на компактах из $U$, мы получаем, что $f_{(K)}$ приближается (с любой точностью) в $V(K)$ конечньпи линейными комбинациями элементов $\left(\Phi_{a}\right)_{(K)}$, $a \notin K$. По теореме 1 для каждого $a \notin \widehat{K}$ имеем $\left(\Phi_{a}\right)_{(K)} \in P_{L V}(\widehat{K}) \subset P_{L V}(K)$. Следовательно, найдется $a \in \widehat{K} \backslash K$, для которой $\Phi_{a} \notin P_{L V}(K)$, что и требовалось.

Остается доказать (iii) $\Rightarrow(\mathrm{i})$. Итак, предположим, что $(L, V)$ удовлетворяет условию $(\mathrm{N})$ (а также $(1)-(4))$. Включение $(\Leftarrow)$ в $(\mathrm{i})$ справедливо по теореме 1 (независимо от условия $(\mathrm{N}))$. Теперь установим $(\Rightarrow)$ в $(\mathrm{i})$. Пусть $F$ замкнуто в $\mathbb{R}^{n}$ и $M_{L V}(F)=E_{L V}(F)$. Необходимо проверить

(a) $G=\mathbb{R}_{\infty}^{n} \backslash F$ связно;

(b) $G=\mathbb{R}_{\infty}^{n} \backslash F$ локально связно в точке $\infty$ 
(поскольку в любой точке $a \in G \backslash\{\infty\}$ множество $G$, очевидно, локально связно).

Докажем (a). Пусть, от противного, $G$ не связно. Тогда $G$ имеет ограниченную (связную, открытую) компоненту $D$ (иначе $G$ была бы связной “через точку" $\infty$ ). Выберем какой-либо шар $B$, содержащий $\bar{D}$, и положим $X=F \cap \bar{B}$. Ясно, что $(\widehat{X} \backslash X) \supset D \neq \varnothing$. Согласно (iii) найдется $a \in \widehat{X} \backslash X$ такое, что $\left(\Phi_{a}\right)_{(X)} \notin$ $P_{L V}(X)$. Поскольку $X \subset F$ и $\widehat{X} \subset \bar{B}$, мы получаем $a \notin F$, откуда $\left(\Phi_{a}\right)_{(F)} \in$ $M_{L V}(F) \backslash E_{L V}(F)$, что противоречит выбору множества $F$.

Будем доказывать (b) также от противного, модифицируя идею Рот (см. [23, c. 155-156]). Поскольку (а) уже доказано, мы можем предположить, что $G$ связно, но не локально связно в точке $\infty$. Из этого предположения (см. замечание 4 ) следует, что найдется шар $B=B(0, R), R>0$, такой, что множество $(G \backslash \bar{B}) \backslash\{\infty\}$ содержит бесконечньй набор (попарно внешних) ограниченных (открытых) компонент связности $D_{m}, m=1,2, \ldots$, удовлетворяющих условию $\operatorname{diam} D_{m} \rightarrow \infty$ при $m \rightarrow \infty$ (подробнее см. [23, гл. IV, раздел 2]). Введем $K_{m}=\widehat{\partial D_{m}} \backslash D_{m}$. Так как $\widehat{K_{m}}=\widehat{\partial D_{m}}$, то по условию $(\mathrm{N})$ для каждого $m$ найдется $a_{m} \in D_{m}$ такое, что $\Phi_{a_{m}} \notin P_{L V}\left(K_{m}\right)$. По лемме 4 мы вправе выбрать последовательность $\left\{a_{m}\right\}$ так, что $a_{m} \rightarrow \infty$ при $m \rightarrow \infty$. Взяв, если это необходимо, подпоследовательность в $\left\{D_{m}, a_{m}\right\}$, мы дополнительно потребуем, чтобы было выполнено $\left|a_{m}\right|>3 R$ и $D_{m} \subset B\left(0,\left|a_{m+1}\right| / 2\right)$ при всех $m \geqslant 1$. Положим

$$
\varepsilon_{m}=\inf \left\{\left\|\Phi_{a_{m}}-p\right\|_{K_{m}}: p \in P_{L V}\left(K_{m}\right)\right\} .
$$

Заметив, что $\varepsilon_{m}>0$, введем $\lambda_{m}=m / \varepsilon_{m}$. Рассуждая как при доказательстве теоремы 1 , мы можем найти полиномы $Q_{m}, m=1,2, \ldots$, для которых ряд

$$
f(x)=\sum_{m=1}^{+\infty}\left(\lambda_{m} \Phi_{a_{m}}(x)-Q_{m}(x)\right)
$$

сходится в $C^{\infty}$ и, следовательно, равномерно на компактах в $\mathbb{R}^{n}$. Последнее означает, что для каждого компакта $K$ найдется $k \in \mathbb{Z}_{+}$такое, что $a_{m} \notin K$ для всех $m \geqslant k$ и

$$
\sum_{m=k}^{+\infty}\left(\lambda_{m} \Phi_{a_{m}}-Q_{m}\right)
$$

сходится равномерно (и в $C^{\infty}$ ) на $K$ в обычном смысле. Осюда $f \in M_{L V}(F)$ и точки $\left\{a_{m}\right\}$ являются единственно возможными особенностями функции $f$ в $\mathbb{R}^{n}$. По предположению $M_{L V}(F)=E_{L V}(F)$, поэтому найдется такая $L$-целая функция $h$, что $f-h \in V(F)$ и $\|f-h\|_{F} \leqslant 1$. Пусть $M=\max \left\{\|f\|_{3} \bar{B} ;\|h\|_{3} \bar{B}\right\}$, так что $M<+\infty$. При всех $m \geqslant 1$ справедлива оценка

$$
\|f-h\|_{F}=\left\|\lambda_{m} \Phi_{a_{m}}-Q_{m}+\sum_{l \neq m}\left(\lambda_{l} \Phi_{a_{l}}-Q_{l}\right)-h\right\|_{F} \leqslant 1 .
$$

Мы утверждаем, что функция

$$
h_{m}=Q_{m}+h-\sum_{l \neq m}\left(\lambda_{l} \Phi_{a_{l}}-Q_{l}\right)
$$


принадлежит $P_{L V}\left(K_{m}\right)$. Действительно, поскольку $K_{m} \subset B\left(0,\left|a_{l}\right| / 2\right)$ при всех $l>m$, то по теореме 1 достаточно будет установить, что для каждого $l=1,2, \ldots, m-1$ справедливо $\Phi_{a_{l}} \in P_{L V}\left(K_{m}\right)$. На самом деле мы докажем, что $\Phi_{a_{l}} \in P_{L V}\left(\widehat{K_{m}}\right)$, показав, что $a_{l} \notin \widehat{K_{m}}$.

Напомним, что все компоненты множества $G \backslash\{\infty\}$ неограничены (так как $G$ связно), поэтому для каждой точки $a_{l}, l<m$, найдется путь $\gamma_{l}$, лежащий вне $\bar{D}_{m}$, с концевыми точками $a_{l}$ и $a_{m+1}$. Этот путь, к примеру, может идти из точки $a_{l}$ к 0 , оставаясь в $D_{l} \cup\left(\partial D_{l} \cap \partial B \backslash F\right) \cup B$, а затем из 0 к $a_{m+1}$, оставаясь в $D_{m+1} \cup\left(\partial D_{m+1} \cap \partial B \backslash F\right) \cup B$. Здесь мы используем тот факт, что (при всех $m$ ) множество $\partial D_{m}$, не являясь подмножеством $F$, должно содержать некоторьй непустой открытый кусок сферы $\partial B$ (иначе $D_{m}$ - ограниченная компонента дополнения $F$, что невозможно). Таким образом, путь $\gamma_{l}$ принадлежит неограниченной компоненте дополнения множества $\partial D_{m}$, т.е. $\gamma_{l} \cap \widehat{K_{m}}=\varnothing$ и $B \cap \widehat{K_{m}}=\varnothing$. Остается воспользоваться теоремой 1.

Теперь покажем, что $K_{m} \subset(F \cup(\partial B))$ для всех $m$. Пусть, от противного, это не так, т.е. найдется точка $b \in K_{m} \backslash F \backslash(\partial B)$. По доказанному $B \cap \widehat{K_{m}}=\varnothing$, поэтому $b$ не лежит в $B$. По предположению $b$ не принадлежит также $\partial B$ и $F$. Таким образом, $b$ должна находиться в одной из компонент $D$ (отличной от $D_{m}$ ) множества $G \backslash \bar{B}$. Ясно, что $D$ может быть только ограниченной (иначе $D$ лежало бы вне $\partial D_{m}$, т.е. и вне $\left.\widehat{K_{m}}\right)$. С другой стороны, если $D$ ограничена, то, рассуждая как и выше (заменяя $D_{l}$ на $D$ и $a_{l}$ на $b$ ), снова получаем противоречие: $b \notin \widehat{K_{m}}$.

Наконец, из оценок

$$
\|f-h\|_{F} \leqslant 1, \quad\|f-h\|_{3 \bar{B}} \leqslant 2 M
$$

и леммы 5 (для $K=K_{m} \subset F \cup \partial B$, т.е. $K \backslash(2 B) \subset F$ ) мы получаем

$$
\|f-h\|_{K_{m}} \leqslant A(2 M+1)
$$

или, эквивалентно,

$$
\left\|\Phi_{a_{m}}-\frac{h_{m}}{\lambda_{m}}\right\|_{K_{m}} \leqslant \frac{A(2 M+1)}{\lambda_{m}}
$$

где $A$ не зависит от $m$.

Из определения $\varepsilon_{m}$ и того, что $h_{m} \in P_{L V}\left(K_{m}\right)$, имеем $\varepsilon_{m} \leqslant A(2 M+1) / \lambda_{m}$ при всех $m \geqslant 1$.

Однако последнее неравенство не может выполняться, так как $\varepsilon_{m} \lambda_{m}=m \rightarrow \infty$ при $m \rightarrow \infty$. Полученное противоречие завершает доказательство теоремы 3 .

4.2. Примеры и следствия. Теперь мы приведем несколько примеров и замечаний, связанных с условием $(\mathrm{N})$.

Обозначим через $B C^{m}\left(\mathbb{R}^{n}\right), m=0,1, \ldots$, пространство всех $m$-раз непрерьвно дифференцируемых функций $f: \mathbb{R}^{n} \rightarrow \mathbb{C}$ c (конечной) нормой

$$
\|f\|_{m}=\max _{|\alpha| \leqslant m} \sup _{x \in \mathbb{R}^{n}}\left|\partial^{\alpha} f(x)\right|
$$

и пусть $C^{m}\left(\mathbb{R}^{n}\right)=\left(B C^{m}\left(\mathbb{R}^{n}\right)\right)_{\text {loc }}$. 
ПримеР 1. Пусть $V=B C^{0}\left(\mathbb{R}^{2}\right)$ и $L=\bar{\partial}=\partial / \partial \bar{z}$ - оператор Коши-Римана в $\mathbb{C}_{z}$. Выполнение условий (1), (2) и (4) проверяется элементарно. Справедливость (3) следует из $[8$, гл. $2, \S 3]$. Для удобства читателя установим справедливость условия (N) (см. [23, гл. $2, \S 3])$.

Пусть $K(K \neq \widehat{K})$ - компакт в $\mathbb{C}$ и $D$-какая-либо компонента из $\widehat{K} \backslash K$. Докажем большее: $\Phi_{a}(z)=1 /(\pi(z-a)) \notin P_{L V}(\partial D)$ для всех $a \in D$. Пусть, от противного, $\Phi_{a}(z) \in P_{L V}(\partial D)$ для некоторого $a \in D$. Тогда найдется последовательность $\left\{p_{m}\right\}$ полиномов комплексного переменного, удовлетворяющая условию $p_{m} \rightarrow \Phi_{a}$ равномерно на $\partial D$ при $m \rightarrow \infty$, что эквивалентно

$$
\left\|(z-a)\left(p_{m}(z)-\Phi_{a}(z)\right)\right\|_{0, \partial D} \rightarrow 0 \text { при } m \rightarrow \infty .
$$

Последнее противоречит принципу максимума модуля в $D$, поскольку

$$
\lim _{z \rightarrow a}(z-a)\left(p_{m}(z)-\Phi_{a}(z)\right)=-\frac{1}{\pi}
$$

(особенность в точке $z=a$ устранима для функции под знаком предела).

Таким образом, из теоремы 2, теоремы 3 и теоремы Мергеляна [4] вытекает результат теоремы (А) (подробности в следуюшем примере).

ПримеР 2. Зафиксируем произвольную положительную радиально симметричную функцию $\omega=\omega(z)=\omega(|z|)$ на плоскости $\mathbb{C}$. Пусть $V$ - пространство функций $f \in C^{0}(\mathbb{C})$, для которых

$$
\|f\|_{\omega}=\sup _{z \in \mathbb{C}}|f(z)| \omega(z)<\infty .
$$

Пространство $V$ (с нормой $\left.\|\cdot\|_{\omega}\right)$ и оператор $L=\bar{\partial}$ удовлетворяют условиям (1), $(2),(3)$ и $(\mathrm{N})$, так как локально норма $\|\cdot\|_{\omega}$ эквивалентна равномерной.

СлЕДСТВИЕ 1. Если вес $\omega$ при некотором $q>0$ удовлетворяет свойству

(a) $\lim _{z \rightarrow \infty} \omega(z)|z|^{-q}=0$,

то для любого замкнутого $F$ в $\mathbb{C}$

(b) $V_{L}(F)=E_{L V}(F) \Longleftrightarrow M_{L V}(F)=E_{L V}(F) \Longleftrightarrow F$ есть РКЛ-множество.

ДокАЗАТЕльСтво. Согласно [6, теорема 4] условие (а) этого следствия равносильно условию (4). Поскольку всегда выполняется $E_{L V}(F) \subset M_{L V}(F) \subset V_{L}(F)$, по теореме 3 нам остается доказать, что если $F$ есть РКЛ-множество, то $V_{L}(F)=$ $M_{L V}(F)$. Последнее вытекает из теоремы 2 и теоремы Мергеляна [4], так как в этом случае для любого замкнутого круга $\bar{B}$ в $\mathbb{C}$ компакт $F \cap \bar{B}$ имеет связное дополнение. Следствие 1 доказано.

Полезно отметить, что в [6, теорема 4] соответствующее условие (а) представляет собой точное условие, при котором справедлива теорема 2 из п. 3.1 (в рамках рассматриваемого примера). Однако в следствии 1 условия (a) и (b) не эквивалентны. Аракелян доказал (см. [23, с. 175]), что (b) остается верным для весов $\omega$ со свойством

$$
\int_{1}^{+\infty} \frac{\log \omega(t)}{t^{3 / 2}} d t<+\infty
$$

Например, таковьм является $\omega(t)=\exp \left(t^{1 / 2-\varepsilon}\right), \varepsilon>0$. 
ПримеР 3. Пусть $V=B C^{1}\left(\mathbb{R}^{n}\right)$ и $L=\Delta, n \geqslant 2$. В работе $[25, \S 7]$ (см. также $[26$, c. 125$])$ доказано, что указанная пара $(L, V)$ удовлетворяет условию $(\mathrm{N})$. Точнее, показано большее: для любой ограниченной области $D$ и $K=\partial D$ выполнено $D \subset N(K)$. Поскольку $(L, V)$ удовлетворяет также (1)-(4) (см. [6] и [25]), имеет место

СЛЕДСТВИЕ 2. При $V=B C^{1}\left(\mathbb{R}^{n}\right)$ u $L=\Delta$ для любого замкнутого множества $F \subset \mathbb{R}^{n}$ следующие утверждения эквивалентнь:

(a) $V_{L}(F)=E_{L V}(F)$;

(b) $F$ есть РКЛ-множество и, кроме того, для каждого иара $B=$ $B(x, \delta)$ имеем $\varkappa\left(B \backslash F^{\circ}\right)=\varkappa(B \backslash F)$

(c) $F$ является РКЛ-множеством и найдутся $k \geqslant 1$ и $A>0$ такие, что $\varkappa\left(B(x, \delta) \backslash F^{\circ}\right) \leqslant A \varkappa(B(x, k \delta) \backslash F)$ для каждого шара $B(x, \delta)$.

(Здесь $\varkappa(\cdot)$ есть $C^{1}$-гармоническая емкость (см. [25]).)

ДокАЗАТЕльство. Аналогично доказательству следствия 1. Заметим только, что из теоремы 2 п. 3.1, из [25, теорема 2] и стандартных аргументов [25, с. 1363] вытекает, что условие $V_{L}(F)=M_{L V}(F)$ эквивалентно емкостным условиям, приведенным в (b) и (c).

ПримеР 4. В этом примере положим $V=B C^{0}\left(\mathbb{R}^{n}\right)$ (с равномерной нормой $\left.\|f\|_{0}=\sup \left\{|f(x)|: x \in \mathbb{R}^{n}\right\}\right)$ и $L=\Delta$ - лапласиан. Хорошо известно, что всякая жорданова область $D$ с гладкой границей $\partial D$ является регулярной для задачи Дирихле, поэтому для $K=\partial D$ имеем $N(K)=\varnothing$. Однако сушествуют также иррегулярные ограниченные области $G, 0 \in G$, для которых при $F=\partial G$ выполнено $\Phi=\Phi_{0} \notin P_{L V}(F)$, т.е. $N(F) \neq \varnothing$. Нетрудно непосредственно установить, что в данном примере условия (1)-(4) вьполнены, однако утверждения (i)-(iii) теоремы 3 нарушаются (например, $\left.P_{L V}(\partial B(0,1))=V(\partial B(0,1))=M_{L V}(\partial B(0,1))\right)$. Если (в предыдуших обозначениях) взять $K$ и $F$ так, чтобы $\widehat{K} \cap \widehat{F}$ содержало не более одной точки, то компакт $K \cup F$ имеет ограниченную компоненту дополнения $D$, не пересекающую $N(K \cup F)$, и компоненту $G$ дополнения, имеющую с $N(K \cup F)$ общие точки.

Полную характеристику $L V$-множеств Аракеляна для этого примера (равномерные гармонические приближения) получил Гардинер (см. [9]).

ПримеР 5. Пусть $L=\partial^{2} / \partial \bar{z}^{2}$ и $V=B C^{0}\left(\mathbb{R}^{2}\right)$. Мы найдем такую аналитическую жорданову кривую $K$, ограничивающую область $D$, для которой $N(K)$ устроено не тривиально: оно не пусто и не совпадает с $D$.

Интересно отметить [27], что для рассматриваемой пары $(L, V)$ найдутся такие аналитические жордановы кривые $F$, для которых $C(F)=P_{L V}(F)$, а также такие, для которых последнее равенство нарушается.

Итак, обозначим через $K$ образ кривой (эллипса) $\left\{z=x+y i \in \mathbb{C}: x^{2}+2 y^{2}=1\right\}$ при отображении $w=1 / z$ и через $D$ - область, ограниченную контуром $K$. Элементарные выкладки показывают, что на $K$ выполняется следуюшее соотношение (уравнение Шварца кривой $K): \bar{w}=w h(w) /\left(4 w^{2}+1\right)$, где $h$ - голоморфная ветвь функции $3+2 \sqrt{2-w^{2}}$ в некоторой окрестности множества $\bar{D}=\widehat{K}$, равная 5 при $w=1$. Пользуясь полярными координатами, нетрудно показать, что приведенное соотношение (Шварца) не вьполняется ни в одной точке области $D$, за искючением точки $w=0$. 
Отметим, что решения уравнения $L u=0$ в какой-либо области $G$ (так называемые бианалитические функиии в $G$ ) имеют вид: $u(w)=h_{1}(w) \bar{w}+h_{0}(w)$, где $h_{0}$ и $h_{1}$ - голоморфные в $G$ функции. В частности, указанные решения являются бианалитическими полиномами, если и только если $h_{0}$ и $h_{1}-$ полиномы комплексного переменного $w$. Далее, если $h_{0}$ и $h_{1}$ голоморфны в окрестности $\widehat{K}$, то функция $u$ (см. выше) принадлежит $P_{L V}(K)$, что вытекает из теоремы Рунге. Поскольку $\Phi(w)=\bar{w} /(\pi w)$, то из вышеприведенного уравнения Шварца для $K$ получаем, что $\pi \Phi(w)=-4 w \bar{w}+h(w)$ на $K$, откуда $0 \notin N(K)$.

Пусть теперь $a \in D \backslash\{0\}$, т.е. $a h(a) \neq \bar{a}\left(4 a^{2}+1\right)$. Используя (на $\left.K\right)$ вместо $\bar{w}$ его выражение из формулы Шварца (в том числе и в формуле для $\Phi(w-a)$ ), а также идею доказательства из примера 1 , получаем, что $a \in N(K)$. Что и требовалось.

В заключение этого пункта мы установим следующее предложение (редукционную теорему для классов функций (струй)).

ПРЕДЛОЖЕНИЕ 1. Пусть пара $(L, V)$ удовлетворяет условиям (1)-(4). Следующие условия на замкнутое множество $F$ в $\mathbb{R}^{n}$ әквивалентны:

(i) справедливо равенство $V_{L}(F)=M_{L V}(F)$;

(ii) для кажсдого иара $B=B(a, r)$ в $\mathbb{R}^{n}$ и любого $\theta \in(0,1)$ имеет место вложсение

$$
V_{L}(\bar{B} \cap F) \subset M_{L V}(\bar{B}(a, \theta r) \cap F) ;
$$

(iii) существует локально конечное покрытие $\left\{B\left(a_{j}, r_{j}\right)\right\}$ множсества $F$ шарами такое, что при каждом $ј$ найдется $k_{j} \geqslant 1$ такое, что

$$
V_{L}\left(\bar{B}\left(a_{j}, k_{j} r_{j}\right) \cap F\right) \subset M_{L V}\left(\bar{B}\left(a_{j}, r_{j}\right) \cap F\right)
$$

Напомним, что если $K$ - компакт, принадлежащий замкнутому множеству $F$ в $\mathbb{R}^{n}$, то каждый элемент $f_{(F), \text { loc }} \in V_{\text {loc }}(F)$ естественным образом определяет элемент $f_{(K)}$ в $V(K)$, так что указанные в предложении 1 вложения пространств определены корректно.

Отметим, что в теореме 4 далее будут рассмотрены такие пары $(L, V)$ (со свойствами (1)-(4)), для которых в предложении 1 можно положить $\theta=1$, а также такие $(L, V)$, для которых (i) $\Rightarrow$ (ii) при $\theta=1$ уже не верно.

ДОКАЗАТЕЛЬСТВО ПРЕДЛОЖЕНИЯ 1. (i) $\Rightarrow$ (ii). Пусть $B=B(a, r)$ и $\theta>0-$ произвольны. Возьмем какую-либо $f_{(\bar{B} \cap F)} \in V_{L}(\bar{B} \cap F)$ с представителем $f \in V$. Выберем $\varphi \in C_{0}^{\infty}(B)$, принимающую значение 1 в некоторой окрестности $\bar{B}(a, \theta r)$. Положим $f_{1}=\mathscr{V}_{\varphi} f$ (см. п. 2.2), $f_{2}=f-f_{1}$. По свойству оператора Витушкина имеем $f_{1} \in V_{\text {loc }} \cap L\left(F^{\circ}\right)$, т.е. согласно (i)

$$
f_{1} \in M_{L V}(F) \subset M_{L V}(\bar{B}(a, \theta r) \cap F)
$$

Но $L f_{2}=0$ в окрестности $\bar{B}(a, \theta r)$, т.е. по теореме $1 f_{2} \in M_{L V}(\bar{B}(a, \theta r) \cap F)$, что и требовалось.

Поскольку (ii) $\Rightarrow$ (iii) очевидно, a (iii) $\Rightarrow$ (i) непосредственно вытекает из теоремы 2, то предложение 1 доказано. 
4.3. Условие $(\mathbf{N})$ и порядок пространства $V$. Пусть $V$ - банахово пространство, удовлетворяюшее условиям (1) и (2) из $\S 2$. Будем говорить, что порядок (әладжости) пространства $V$ не меньше $m(\operatorname{Ord}(V) \geqslant m), m=0,1, \ldots$, если

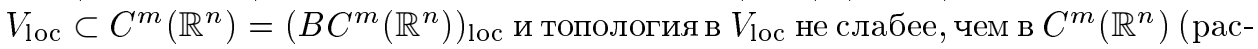
сматриваем $B C^{m}\left(\mathbb{R}^{n}\right)$ как подпространство в $\left(C_{0}^{\infty}\right)^{*}$, удовлетворяюшее (1) и (2)). Если $\operatorname{Ord}(V) \geqslant 0$, то определим $\operatorname{Ord}(V)=\sup \{m: \operatorname{Ord}(V) \geqslant m\}$.

В работе А.Г. О'Фаррелла [28] приводится естественное конструктивное определение $\operatorname{Ord}(V)$ для широкого класса так называемых "симметрических конкретных банаховых пространств" (SCBS), а также (основанная на этом определении) абстрактная теорема вложения соболевского типа.

Пусть $L$ - эллиптический оператор порядка $r \geqslant 1$ в $\mathbb{R}^{n}$ (см. §2). При $n=2$ нам не потребуется никаких дополнительных ограничений на $L$, но при $n \geqslant 3$ нам придется рассматривать операторы $L$ со следуюшим символом:

$$
L(\xi)=P_{2}(\xi) Q_{r-2}(\xi), \quad \xi \in \mathbb{R}^{n},
$$

где $P_{2}$ - некоторый однородный (эллиптический) полином порядка 2 с вещественньми коэффициентами (так что $P_{2}$ знакоопределен в $\mathbb{R}^{n} \backslash\{0\}$ ) и $Q_{r-2}-$ однородный полином порядка $r-2$.

ПРЕДЛОЖЕНИЕ 2. Пусть L удовлетворяет условию (7) (в частности, никакого дополнительного ограничения при $n=2)$, а пара $(L, V)$ - условиям (1) и (2). Если $\operatorname{Ord}(V) \geqslant r-1$, то $(L, V)$ удовлетворяет условию $(\mathrm{N})$.

Отметим, что формулировка условия $(\mathrm{N})$ не использует условия (3) и (4).

СлеДСтвИЕ 3. Если $(L, V)$ удовлетворяет условиям (1)-(4), (7) $и \operatorname{Ord}(V) \geqslant$ $r-1, m o$

$$
M_{L V}(F)=E_{L V}(F) \Longleftrightarrow F \text { есть РКЛ-множество. }
$$

Вопрос о том, справедливо ли предложение 2 без требования условия (7), остается открытым. Представляет интерес также и следующий вопрос: существует ли пара $(L, V)$, удовлетворяюшая условиям (1)-(4), для которой $\operatorname{Ord}(V)<$ $\operatorname{Ord}(L)-1$, но при этом утверждение (8) остается верным?

ДоКАЗАТЕЛЬСТВо ПРЕДЛОЖЕНИЯ 2. Аналогично примерам 1 и 3 мы установим, что (в условиях предложения 2) если $D$ - ограниченная область в $\mathbb{R}^{n}$, то для $K=\partial D$ имеем $D \subset N(K)$. Пусть, от противного, найдется $a \in D$ и последовательность $\left\{p_{m}^{a}\right\}$ таких $L$-аналитических полиномов, что $\left\|\Phi_{a}-p_{m}^{a}\right\|_{K} \rightarrow 0$ при $m \rightarrow \infty$. Это в точности означает, что найдется $f^{a} \in V$, которая совпадает с $\Phi_{a}$ в некоторой окрестности $U^{a}$ множества $K$, и такие $h_{m}^{a} \in V$, равные $p_{m}^{a}$ в каких-то своих окрестностях $U_{m}^{a}$ компакта $K, U_{m}^{a} \subset U^{a}, m=1,2, \ldots$, что $\left\|f^{a}-h_{m}^{a}\right\| \rightarrow 0$ при $m \rightarrow \infty$.

Выберем некоторьй шар $B$, содержащий $K$. Так как $\operatorname{Ord}(V) \geqslant r-1$, то мы знаем, что $f^{a}$ и $h_{m}^{a}$ принадлежат $C^{r-1}(\bar{B})$, причем

$$
\left\|f^{a}-h_{m}^{a}\right\|_{r-1, \bar{B}} \rightarrow 0 \text { при } m \rightarrow \infty .
$$

Пользуясь подходящей (линейной невырожденной) заменой переменных в $\mathbb{R}^{n}$, мы можем свести нашу ситуацию к одной из следуюших: при $n=2$ оператор $L$ 
имеет форму $L u=\bar{\partial} L_{r-1} u$, а при $n \geqslant 3-$ форму $L u=\Delta L_{r-2} u$, где $L_{r-1}$ и $L_{r-2}$ - некоторые однородные дифференциальные операторы с постоянными коэффициентами порядка $r-1$ и $r-2$, соответственно. Очевидно, что класс $C^{r-1}\left(\mathbb{R}^{n}\right)$ и класс всех полиномов в $\mathbb{R}^{n}$ (от действительных переменных) инвариантны относительно указанной замены переменных и, более того, имеется естественное соответствие между решениями исходного уравнения и нового (которое снова запишем как $L u=0)$. Например, исходное $\Phi_{a}$ соответствует новому $k \Phi_{b}$, где $k \in \mathbb{R} \backslash\{0\}$ и $\Phi_{b}$ - соответствующий сдвиг нового фундаментального решения. Следовательно, соответствующее (новое) свойство (9) будет выполняться для нового $L$ и нового (эллипсоида) $B$.

Действуя оператором $L_{r-1}$ (при $n=2$ ) или $L_{r-2}$ (если $n \geqslant 3$ ) на разность, записанную внутри нормы в (9), мы получим

$$
\begin{gathered}
\left\|L_{r-1} f^{a}-L_{r-1} h_{m}^{a}\right\|_{0, \bar{B}} \rightarrow 0 \text { при } m \rightarrow \infty \quad(n=2), \\
\left\|L_{r-2} f^{a}-L_{r-2} h_{m}^{a}\right\|_{1, \bar{B}} \rightarrow 0 \text { при } m \rightarrow \infty \quad(n \geqslant 3) .
\end{gathered}
$$

Поскольку $L_{r-1} h_{m}^{a}, n=2$ (соответственно, $L_{r-2} h_{m}^{a}$ при $\left.n \geqslant 3\right)$, совпадает с некоторым комплексным (соответственно, гармоническим при $n \geqslant 3$ ) полиномом в $U_{m}^{a}$ и $L_{r-1} f^{a}$ (соответственно, $L_{r-2} f^{a}$ для $n \geqslant 3$ ) пропорционально в $U^{a}$ фундаментальному решению для оператора $\bar{\partial}$ (соответственно, $\Delta$ для $n \geqslant 3$ ), мы получаем нужное противоречие с фактами, изложенньми в примерах 1 и 3 п. 4.2. Доказательство завершено.

В качестве приложения доказанных вьше утверждений мы получаем следующие аналоги теорем Нерсесяна [24] (см. также [29]) и Аракеляна [3].

ТЕОРемА 4. Пусть L (порядка $r$ ) удовлетворяет свойству $(7), F$ - замкнутое подмножсество в $\mathbb{R}^{n}$.

(i) При $V=\operatorname{lip}\left(s, \mathbb{R}^{n}\right)$, әде $s \in(r-1, r)$ (см. [16]), для выполнения равенства $V_{L}(F)=M_{L V}(F)$ необходимо и достаточно, чтоби существовала константа $A \in(0,+\infty)$ такая, что для всякого шара $B$ в $\mathbb{R}^{n}$

$$
M_{*}^{n-r+s}\left(B \backslash F^{\circ}\right) \leqslant A M^{n-r+s}(B \backslash F) .
$$

(ii) $\Pi p u V=B C^{m}\left(\mathbb{R}^{n}\right), m=r, r+1, \ldots$, uлu $V=\operatorname{lip}\left(s, \mathbb{R}^{n}\right), s>r, s \notin \mathbb{Z}, \partial \Omega я$ выполнения равенства $V_{L}(F)=M_{L V}(F)$ необходимо и достаточно, чтобь $F^{\circ}$ бълло всюду плотно в $F$.

(iii) Для кажсдого из упомянутых в (i) или (ii) пространств $V$ равенство $V_{L}(F)=E_{L V}(F)$ справедливо, если и только если $V_{L}(F)=M_{L V}(F)$ и (одновременно) F является РКЛ-множеством.

Здесь $M^{n-r+s}(\cdot)$ и $M_{*}^{n-r+s}(\cdot)$ есть обхват по Хаусдорфу и нижний обхват по Хаусдорфу порядка $n-r+s$, соответственно (см. [21]).

ДокАЗАТЕльство. (i) Необходимость (10) проверяется так же, как в [21, с. 187] (см. также [25, с. 1363]). Для доказательства достаточности нужно установить, что из (10) вытекает, что для каждого замкнутого шара $\bar{B}_{1}$ при $F_{1}=F \cap \bar{B}_{1}$ имеем $V_{L}\left(F_{1}\right)=M_{L V}\left(F_{1}\right)$ (априори не известно, что $(10)$ справедливо для $F_{1}$ вместо $F)$. Однако легко проверить, что (10) влечет

$$
M_{*}^{n-r+s}\left(B \backslash F_{1}^{\circ}\right) \leqslant A_{1} M^{n-r+s}\left(2 B \backslash F_{1}\right)
$$


для всех шаров $B$, где $A_{1}>0$ не зависит от $B$. Наконец, доказательство теоремы 2 в [21] остается справедливым (практически без изменений), если вместо (10) мы всюду потребуем (11), что дает $V_{L}\left(F_{1}\right)=M_{L V}\left(F_{1}\right)$. Остается воспользоваться предложением 1 . Отметим, что в этом случае предложение 1 верно и при $\theta=1$.

(ii) Необходимость устанавливается аналогично [26, следствие 2.9]. Достаточность следует из [21, теорема 1], [30] и предложения 1. Здесь, однако, при $\theta=1$ уже не верно, что (i) $\Rightarrow$ (ii) в предложении 1 . Достаточно рассмотреть $F=\left\{x \in \mathbb{R}^{n}\right.$ : $|x| \geqslant 1\}$ и $B(a, r)=B(0,1)$.

(iii) Непосредственно следует из (i), (ii), теоремы 3 и предложения 2. Теорема доказана.

В заключение отметим, что так же, как в классическом случае $L=\bar{\partial}, V=$ $B C(\mathbb{C})$ (см. [23, гл. 4]), большинство результатов этой работы можно обобщить (с необходимыми очевидными изменениями) на случай, когда пространство $V$ определено не на всем $\mathbb{R}^{n}$, а на некоторой его области. Эти обобшения и их приложения к теории граничных значений $L$-аналитических функций мы планируем изложить в последуюшей работе.

\section{Список литературы}

1. Runge C. Zur theorie der eindeutigen analytischen funktionen // Acta Math. 1885. V. 6 . P. 228-244.

2. Roth A. Approximationseigenschaften und strahlengrenzwerte meromorpher und ganzer funktionen // Comment. Math. Helv. 1938. V. 11. P. 77-125.

3. Аракелян Н. У. Равномерная аппроксимация на замкнутых множествах целыми функциями // Изв. АН СССР. Сер. матем. 1964. Т. 28. № 5. С. 1187-1206.

4. Мергелян С. Н. Равномерноеприближение функций комплексного переменного // УМН. 1952. T. 7. № 2 (48). C. 31-122.

5. O'Farrell A. G. T-invariance // Proc. Roy. Irish Acad. Sect. A. 1992. V. 92. № 2. P. 185-203.

6. Paramonov P. V., Verdera J. Approximation by solutions of elliptic equations on closed subsets of Euclidean space // Math. Scand. 1994. V. 74. P. 249-259.

7. Dufresnoy A., Gauthier P.M., Ow W. H. Uniform approximation on closed sets by solutions of elliptic partial differential equations // Complex Variables Theory Appl. 1986. V. 6. P. 235-247.

8. Витушкин А. Г. Аналитическая емкость множеств в задачах теории приближений // УМH. 1967. T. 22. №6. С. 141-199.

9. Gardiner S. J. Harmonic Approximation. London Mathematical Society Lecture Notes Series. V. 221. Cambridge: Cambridge Univ. Press, 1995.

10. Lax $P$. A stability theorem for abstract differential equations and its applications to the study of local behaviour of solutions of elliptic equations // Comm. Pure Appl. Math. 1956. V. 9. P. $747-766$.

11. Malgrange $B$. Existence et approximation des solutions des equations aux dérivées partielles et des equations de convolution // Ann. Inst. Fourier (Grenoble). 1955-56. V. 6. P. 271-355.

12. Fariña J. C. Lipschitz approximation on closed sets // J. Anal. Math. 1991. V. 57. P. $152-171$.

13. Bonilla A., Fariña J. C. Meromorphic and holomorphic approximation in $C^{m}$-norms // J. Math. Anal. Appl. 1994. V. 181. P. 132-149.

14. Bonilla A., Fariña J. C. Meromorphic and entire approximation in BMO-norm // J. Approx. Theory. 1994. V. 76. P. 203-218.

15. Bagby T., Gauthier P. M. Uniform approximation by global harmonic functions // Approximation by Solutions of Partial Differential Equations. NATO ASI Series. Dordrecht: Kluwer Acad. Publ., 1992. P. 15-26. 
16. Verdera J. Removability, capacity and approximation // Complex Potential Theory. NATO ASI Series. Dordrecht: Kluwer Acad. Publ., 1993. P. 419-473.

17. Hörmander L. Linear Partial Differential Operator. Berlin: Springer-Verlag, 1963.

18. Husain T. The Open Mapping and Closed Graph Theorems in Topological Vector Spaces. London: Oxford Univ. Press, 1965.

19. Reed M., Simon B. Methods of Modern Mathematical Physics. V. I. New York: Academic Press, 1972.

20. Стейн И. М. Сингулярные интегралы и дифференциальные свойства функций. М.: Мир, 1973.

21. Verdera J. $C^{m}$ approximation by solutions of elliptic equations, and Calderón-Zygmund operators // Duke Math. J. 1987. V. 55. P. 157-187.

22. Келдиш M.В., Лаврентьев М. А. Sur une probleme de M. Carleman // Докл. АН CСCP. 1939. T. 23. № 8 . C. 746-748.

23. Гайер Д. Лекции по теории аппроксимации в комплексной области. М.: Мир, 1986.

24. Нерсесян A. A. Равномерная и касательная аппроксимация мероморфньми функциями // Изв. АН Арм. ССР. Сер. матем. 1972. Т. 7. С. 405-412.

25. Парамонов П. В. О гармонических аппроксимациях в $C^{1}$-норме // Матем. сб. 1990. T. 181. № 10. С. 1341-1365.

26. Парамонов П.В. $C^{m}$-приближения гармоническими полиномами на компактных множествах в $\mathbb{R}^{n}$ // Матем. сб. 1993. Т. 184. № 2. С. 105-128.

27. Федоровский $K$. Ю. О равномерных приближениях функций $n$-аналитическими полиномами на спрямляемых контурах в $\mathbb{C} / /$ Матем. заметки. 1996. Т. 59. № 4. С. 604-610.

28. O'Farrell A. G. The order of a symmetric concrete space // Proc. Roy. Irish Acad. Sect. A. 1988. V. 88. № 1. P. 39-48.

29. Boivin A., Verdera J. Approximation par fonctions holomorphes dans les espaces $L^{p}, \operatorname{Lip}_{\alpha}$ et BMO // Indiana Univ. Math. J. 1991. V. 40. P. 393-418.

30. O'Farrell A. G. Rational approximation in Lipschitz norms - II // Proc. Roy. Irish Acad. Sect. A. 1979. V. 79. № 11. P. 103-114.

University of Western Ontario, London, Canada;

Московский государственный университет им. М.В. Ломоносова

Поступила в редакцию 23.06 .1997 